\title{
Supporting Information for: Significant Effect of Surfactant Adsorption Layer Thickness in Equilibrium Foam Films
}

\author{
Mengsu Peng, Timothy T. Duignan, Anh V. Nguyen* \\ School of Chemical Engineering, University of Queensland, Brisbane, Queensland 4072, Australia \\ *Correspondence: anh.nguyen@eng.uq.edu.au
}

\section{Calculate the Hamaker constant}

In addition to the EDL pressure, the total disjoining pressure, $\Pi(H)$ as measured by the film balance technique also includes the van d Waals disjoining pressure, $\Pi_{v d W}(H)$ as per the DLVO theory. Here we follow an advanced algorithm used by our group to calculate the van der Waals pressure employing the multilayer (air-adsorption layer-water) dispersion theory ${ }^{1}$. We have:

$$
A_{H}=-\frac{3 k_{B} T}{2} \sum_{n=0}^{\infty} \cdot \int_{x_{n}}^{\infty} x \ln \left[\left\{1-\mathfrak{J}\left(i \omega_{n}\right) e^{-x}\right\}\left\{1-\overline{\mathfrak{J}}\left(i \omega_{n}\right) e^{-x}\right\}\right] d x
$$

where $x$ is the dummy variable, $k_{\mathrm{B}}$ is the Boltzmann constant, and $n$ is the summation index. The prime against the summation indicates that the zero-frequency $(n=0)$ term is divided by 2. The Matsubara (sampling, discrete equally spaced) frequencies are described by $\omega_{n}=2 n \pi k_{B} T / \hbar$ where $\hbar$ is the Planck constant (divided by $2 \pi$ ), $i \omega_{n}$ represents the imaginary frequencies. $x_{n}=2 h \omega_{n} \sqrt{\varepsilon_{m}\left(i \omega_{n}\right)} / c$, where $c$ is the speed of light and $\varepsilon_{m}\left(i \omega_{n}\right)$ describes the dielectric susceptibility of a material $m$, which refers to the central material across which the layered structures interact (i.e., the water phase in this paper). $\sqrt{\mathfrak{I}}$ and $\sqrt{\mathfrak{I}}$ describe the diamagnetic reflection coefficients and the dielectric reflection coefficients of a photon passing through the adsorption layer and water phase from one side of a foam film to another, respectively. By considering the surfactant adsorption layer $(S)$ with thickness $d$ between air $(A)$ and the water phase $(W)$, we have the following expression:

$$
\sqrt{\mathfrak{I}\left(i \omega_{n}\right)}=\frac{\Delta_{W S}+\Delta_{S A} \exp \left[-x d s_{S} /(H q)\right]}{1+\Delta_{W S} \Delta_{S A} \exp \left[-x d s_{S} /(H q)\right]}
$$


In Eq.S2, $\Delta_{W S}$ and $\Delta_{S A}$ are the diamagnetic reflection coefficients of a photon passing across a single interface between two materials, and are given by:

$$
\Delta_{j k}=\frac{s_{j}-s_{k}}{s_{j}+s_{k}}
$$

where the subscripts $j$ and $k$ refer to the two materials, and the retardation coefficients, $\mathrm{s} j$, are defined by the following generic expression:

$$
s_{j}=\sqrt{q^{2}-1+\varepsilon_{j}\left(i \omega_{n}\right) / \varepsilon_{m}\left(i \omega_{n}\right)}
$$

where $\varepsilon_{j}\left(i \omega_{n}\right)$ describes dielectric susceptibility of a material $j$, which refers to the particular material of each layer, i.e., water or adsorption layer in this paper and the dielectric susceptibility of air, $\varepsilon_{A}\left(i \omega_{n}\right)=1$. In Eq.S4, parameter $q$ is defined as follows:

$$
q=\frac{x c}{2 \omega_{n} h \sqrt{\varepsilon_{m}\left(i \omega_{n}\right)}}
$$

$\sqrt{\overline{\mathfrak{I}}}$ in Eq.S1 can be calculated using Eq.S2 with symbol $\Delta$ being replaced by $\bar{\Delta}$, which are the dielectric reflection coefficients of a photon passing across a single interface between the two materials, and is given by:

$$
\bar{\Delta}_{j k}=\frac{s_{j} \varepsilon_{j}\left(i \omega_{n}\right)-s_{k} \varepsilon_{k}\left(i \omega_{n}\right)}{s_{j} \varepsilon_{j}\left(i \omega_{n}\right)+s_{k} \varepsilon_{k}\left(i \omega_{n}\right)}
$$

The numerical calculation of Eq.S1 was performed using the Gauss-Laguerre quadrature. ${ }^{2}$ The computational results were obtained using the values of the zeros of the Laguerre polynomial and the respective weights of the $30^{\text {th }}$ order Gauss-Laguerre quadrature. The computation of the outer summation included at least 3000 terms for satisfactory convergence and accuracy.

\section{The effect of non-electrostatic forces on the disjoining pressure}

There may be some concerns about whether the ion concentration at the middle plane can be calculated by the following equation: 


$$
\Pi_{e d l}(H)=k_{B} T \sum n_{i \infty}\left[\exp \left(-\frac{z_{i} e \psi_{m}}{k_{B} T}\right)-1\right]
$$

As the MPB model includes multiple energy terms, and the EDL component of the disjoining pressure should be calculated from the concentration of ions in the middle plane using a more rigorous equation:

$$
\Pi_{e d l}(H)=k_{B} T \sum n_{i \infty}\left\{\exp \left[-\frac{1}{k_{B} T}\left(z_{i} e \psi+G_{i m}+G_{i t}\right)_{x=H / 2}\right]-1\right\}
$$

However, Eq. S7 and Eq. S8 give practically the same results for all the films we have discussed in this paper. This is because the electrostatic force is the only long-range force which affects the ion concentration far from the interface, i.e. at the middle plane. Figure S1 shows the significant decrease of non-electrostatic energies as they approach the middle plane in a film formed by $7 \mathrm{mM}$ SDS and $0.5 \mathrm{mM} \mathrm{NaCl}$.

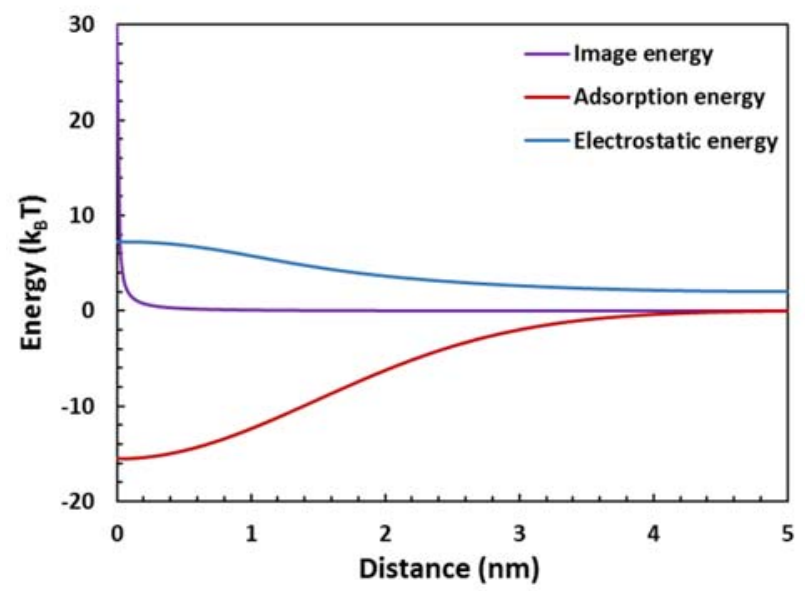

Figure S1. The reduction of energies according to the distance away from the interface predicted by the MPB model. Only the electrostatic energy has a non-zero value after $5 \mathrm{~nm}$.

We can see that the adsorption energy and image energy reduced to almost zero at $5 \mathrm{~nm}$ away for the interface while the electrostatic energy is more than $2 \mathrm{k}_{\mathrm{B}} \mathrm{T}$, so other forces will not affect the ion concentration at the middle plane of the thin liquid film. As other energy terms are ignorable compared to the electrostatic energy under this condition, Eq. S7 is accurate to account for all experimental results we used in this work. Although we apply the MPB equation to calculate the electrostatic potential, we don't need to consider force other than the electrostatic one to modelling the disjoining pressure. 


\section{The effect of the film thickness on the ion distribution}

Figure S2 shows the density distribution of ions from MD simulation with different thickness of the water layer between adsorption layers. The ion distribution does not change significantly with the thickness of the water layer. However, the overlapping of adsorption layers will cause some trouble if the water layer is too thin (e.g. $5 \mathrm{~nm})$. We use a $10 \mathrm{~nm}$ water layer for the best simulation efficiency since $30 \mathrm{~nm}$ water layer is not necessary.
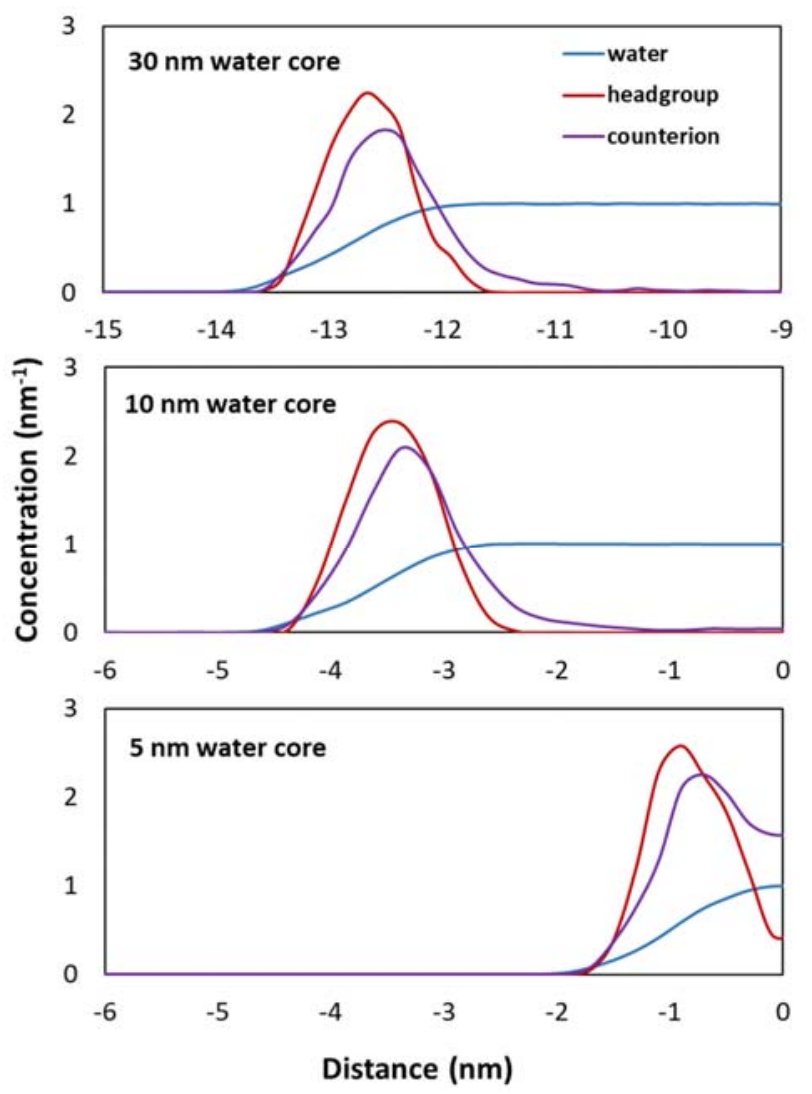

Figure S2. The density distribution of water, headgroup and counterion from MD. The simulations containing 77 SDS molecules at each vacuum-water interface were performed with 30,10 , and $5 \mathrm{~nm}$ water layer in between.

\section{The effect of film thickness on the surfactant adsorption}

Figure S3 shows the effect of film thickness on surfactant adsorption and surface potential profile of the film formed by $7 \mathrm{mM}$ SDS and $0.5 \mathrm{mM} \mathrm{NaCl}$. There are some tiny changes of the adsorption at $\mathrm{x}>3 \mathrm{~nm}$ when a $10 \mathrm{~nm}$ film is formed compared to the free 
interface. However, those changes are very small, and there is no observable change to the potential profile close to the interface of the thin film.
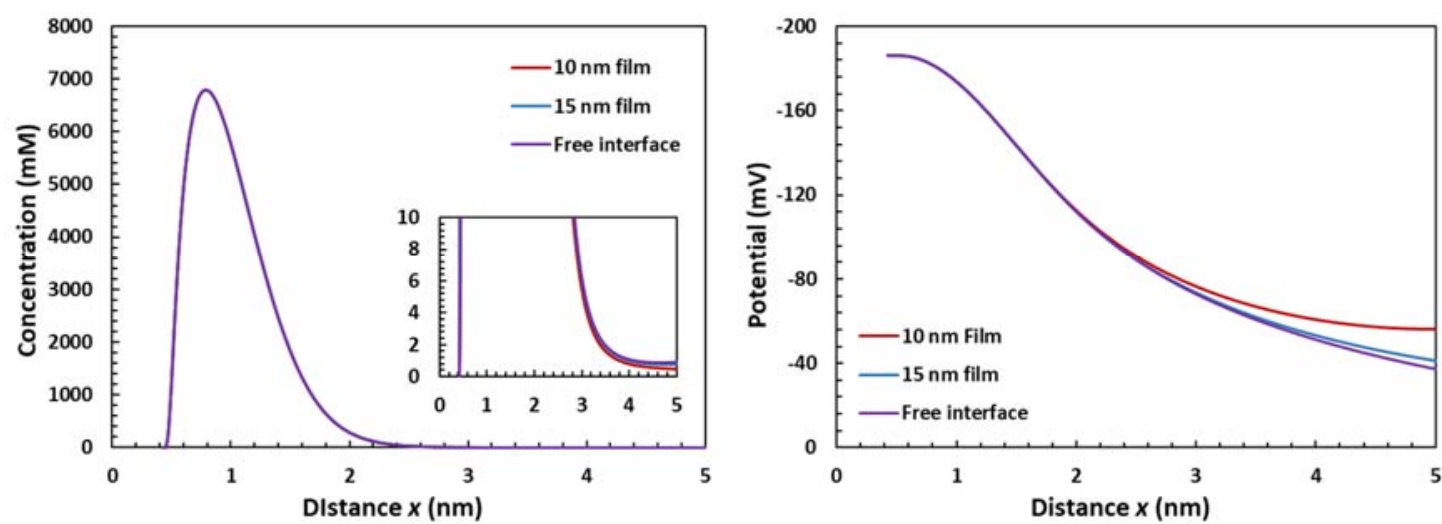

Figure S3. The surfactant concentration (left) and potential (right) change according to film thickness for the thin film formed by $7 \mathrm{mM}$ SDS and $0.5 \mathrm{mM} \mathrm{NaCl}$. The change of adsorption is tiny so that the surfactant concentration profile form three different thickness overlaps.

\section{Calculate potential profiles and the disjoining pressure using classical approaches}

In the case of classical approaches, the middle plane potential, $\psi_{m}$, can be numerically obtained from the classical Poison-Boltzmann (PB) equation. It is described in terms of the electrostatic potential $\psi$ in the film as follows (for a $z: z$ electrolyte with the number concentration, $n_{\infty}$, in the bulk):

$$
\frac{d^{2} \psi}{d x^{2}}=\frac{2 z n_{\infty} e}{\varepsilon \varepsilon_{0}} \sinh \left(\frac{z e \psi}{k_{B} T}\right)
$$

The boundary condition at the film middle plane due to symmetry gives $d \psi / d x=0$ at $x=H / 2$. Another boundary condition is that the potential at the beginning of the diffuse layer (i.e. 1.1 $\mathrm{nm}$ ) as a constant, i.e., $\psi_{0}=-80 \mathrm{mV},-200 \mathrm{mV}$, or $-160 \mathrm{mV}$ (for the case of the adsorption model, this value was taken from the literature ${ }^{3}$ ), where $\psi_{0}$ is the surface potential evaluated at the boundary between the diffuse layer and the adsorption layer. Solving Eq. S9 is straightforward. The numerical solution provides the electrostatic profile and the disjoining pressure can be obtained by Eq.1 with the middle plane potential. 
Some people may argue that the potential at the beginning of the diffuse layer may not be a constant. In this case we can have another boundary condition established from the charge neutrality condition, i.e., the surface charge $Q$ is balanced by the charge of the diffuse layer in the film. The latter can be derived from the first integration of Eq. S9, giving

$$
Q^{2}=4 n_{\infty} \varepsilon \varepsilon_{0} k_{B} T\left[\cosh \left(\frac{z e \psi_{0}}{k_{B} T}\right)-\cosh \left(\frac{z e \psi_{m}}{k_{B} T}\right)\right]
$$

Assuming this charge is constant we can get the boundary condition called constant charge.

In fact, a more realistic boundary considers the change of the charge alters by the surfactant adsorption: the surface charge $Q$ in Eq. S10 can be linked with the adsorption of surfactant and counterion molecules which is described as follows:

$$
Q= \pm z e\left(\Gamma_{1}-\Gamma_{2}\right)
$$

where $\Gamma_{1}$ and $\Gamma_{2}$ denote the surface excess of surfactant ions (denoted by the subscript " 1 ”) and counterions (denoted by the subscript "2"). Coions do not appear in the adsorption layer because of strong electrostatic repulsion. For the adsorption model used in this paper it assumes counterion facilitates the adsorption of surfactant but does not move into the adsorption layer. We have the following equations to account for $\Gamma_{1}$ and $\Gamma_{2}$ :

$$
\begin{aligned}
& {\left[K_{1}+K_{2} n_{2 s}\right] n_{1 s}=\frac{\Gamma_{1}}{\Gamma_{\infty}-\Gamma_{1}} \exp \left[-2 \beta\left(\frac{\Gamma_{1}}{\Gamma_{\infty}}\right)\right]} \\
& \frac{\Gamma_{2}}{\Gamma_{1}}=\frac{K_{2} n_{2 s}}{K_{1}+K_{2} n_{2 s}} \\
& n_{i s}=n_{i \infty} \exp \left(\frac{z e\left|\psi_{0}\right|}{k_{B} T}\right) \text { with } i=1 \text { and } 2
\end{aligned}
$$

where $K_{1}$ and $K_{2}$ are the equilibrium adsorption constants of surfactant ions and counterions, respectively, $\beta$ is the intermolecular interaction constant ( $\beta=0$ yields the Langmuir isotherm), and $\Gamma_{\infty}$ is the maximum surface excess. Eq. S14 relates the surface concentration with the bulk concentration for both surfactant ions and counterions. Eq. S9-S11 can be applied for different adsorption models. The main equation Eq. S9 with the two boundary conditions (one of them is Eq. S10) is numerically solved by the collocation method using the "bvp4c" Solver of 
MATLAB. The initial guess to start the iteration uses the Debye-Hückel analytical solution to the linearized PB equation.

However, in practice, three different boundary conditions give very similar results for film thicker than $10 \mathrm{~nm}$. For convenience, all calculations for classical approaches only use the constant potential condition.

\section{References}

1. Parsegian, V. A.; Ninham, B. W., Van Der Waals Forces in Many-Layered Structures: Generalizations of the Lifshitz Result for Two Semi-Infinite Media. J. Theor. Biol. 1973, 38, 101-109.

2. Nguyen, A. V.; Schulze, H. J., Colloidal Science of Flotation; Marcel Dekker: New York, 2004; Vol. 118.

3. Kralchevsky, P.; Danov, K.; Broze, G.; Mehreteab, A., Thermodynamics of Ionic Surfactant Adsorption with Account for the Counterion Binding: Effect of Salts of Various Valency. Langmuir 1999, 15, 2351-2365. 